\title{
Philosophiques
}

\section{Les deux libéralismes de Charles Taylor : le Québec et le Canada}

\section{Pierre-Yves Bonin}

Volume 22, numéro 1, printemps 1995

URI : https://id.erudit.org/iderudit/027305ar

DOI : https://doi.org/10.7202/027305ar

Aller au sommaire du numéro

Éditeur(s)

Société de philosophie du Québec

ISSN

0316-2923 (imprimé)

1492-1391 (numérique)

Découvrir la revue

Citer cet article

Bonin, P.-Y. (1995). Les deux libéralismes de Charles Taylor : le Québec et le Canada. Philosophiques, 22(1), 3-20. https://doi.org/10.7202/027305ar
Résumé de l'article

Dans de récents travaux, Charles Taylor distingue deux types de libéralisme, qu'il associe respectivement au Canada et au Québec. Le premier se caractérise par l'adhésion à la règle de neutralité de I'État, tandis que le second impose moins de limites à l'intervention de l'État. Parce qu'il serait moins

" homogénéisant ", Taylor se prononce en faveur du second type. Plusieurs éléments de l'analyse de Taylor me semblent faux ou erronés. Premièrement, la distinction entre les deux types de libéralisme n'est pas toujours claire. Deuxièmement, le type de libéralisme que Taylor favorise n'est pas du tout acceptable. Troisièmement, je ne crois pas que le Canada et le Québec adhèrent à des types différents de libéralisme. 


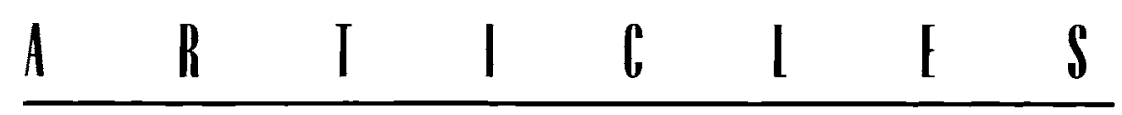

\title{
LES DEUX LIBÉRALISMES DE CHARLES TAYIOR: IE QUUÉBEC ET IE CANADA
}

\author{
par \\ Pierre-Yves Bonin
}

\begin{abstract}
RÉsumÉ : Dans de récents travaux, Charles Taylor distingue deux types de libéralisme, quil associe respectivement au Canada et au Québec. Le premier se caractérise par l'adhésion à la régle de neutralité de l'Etat, tandis que le second impose moins de limites à linter vention de l'Etat. Parce qu'il serait moins « homogénéisant», Taylor se prononce en faveur du second type. Plusieurséléments de l'analyse de Taylorme semblent faux ou erronés. Premierement, la distinction entre les deux types de libéralisme n'est pas toujours claire. Deuxièmement. le type de libéralisme que Taylor favorise n'est pas du tout acceptable. Troisièmement, je ne crois pas que le Canada et le Québec adherent à des types différents de libéralisme.
\end{abstract}

ABSTRACT: In his recent works, Charles Taylor distinguishes between two forms of liberalism, which are respectively associated with Canada and Québec. The first one is characterized by its support for the neutrality of the State, while the second imposes almost no restriction to the intervention of the State. Because it is less « homogenizing», Taylor favors the second form. Many parts of Taylor's analysis seems to me to be false or erroneous. First, the distinction between the two liberalisms is not at all clear. Second, the liberalism that Taylor favors is not acceptable. Third, it is not true that Québec and Canada adhere to different forms of liberalism.

Les sociétés qui n'imposent pas de force une religion ou une conception du bien et du bonheur se caractérisent toutes par la cohabitation, plus ou moins harmonieuse, d'une variété de modes de vie, de cultures et de religions. 
Les membres des groupes et des cultures minoritaires déploient des efforts considérables pour faire reconnaître et respecter leur identité, tandis que les groupes les plus forts cherchent à influencer les politiques de l'État en leur faveur.

Ces luttes pour la reconnaissance et l'affirmation de son groupe social ou culturel ne constituent pas un phénomène récent. Hier, elles mettaient aux prises les majorités religieuses, racistes et sexistes, et les différentes sectes catholiques et protestantes, les Afro-américains, les femmes, les partisans de l'amour libre, etc. Aujourd'hui, les principaux protagonistes sont, d'un côté, les majorités « morales » ou « communautariennes » et, de l'autre, les homosexuels, les autochtones, les minorités ethniques et culturelles, etc.

Dans ces conflits, les libéraux ont toujours pris le parti de la tolérance et du respect des différences, milité pour une plus grande ouverture envers les cultures étrangères et favorisé une plus grande compassion à l'égard des marginaux et des groupes minoritaires. Animés par un profond respect de l'autonomie des individus, les libéraux ont d'abord demandé qu'une fin soit mise aux persécutions et à l'intégration forcée des groupes minoritaires. La répression des minorités se pratiquait alors sur une si vaste échelle qu'un objectif aussi limité que la tolérance représentait déjà un défi énorme. Forts de leurs premiers succès et s'appuyant sur les effets bénéfiques d'une libéralisation des mœurs, les libéraux ont intensifié leur lutte, dénoncé toutes les formes de discrimination et revendiqué rien de moins que l'égalité de tous et de toutes devant la loi et le pouvoir politique. Ce programme de revendications trouve aujourd'hui son point culminant dans la défense et la promotion de la neutralité de l'État à l'égard de tous les groupes sociaux ou culturels. Pour cette nouvelle génération de libéraux, l'État devrait non seulement tolérer les différences, mais ne jamais chercher à favoriser un groupe plutôt qu'un autre, que ce groupe soit majoritaire ou minoritaire.

Ces récents développements de la philosophie politique libérale ne plaisent pas à tous et plusieurs, même parmi les libéraux, sont d'avis qu'il est temps de faire marche arrière ${ }^{\mathrm{I}}$. Le philosophe communautarien Charles Taylor fait partie de ces révisionnistes. Dans un essai et deux articles parus

I. Alistair MacIntyre, After Virtue, Notre Dame, University of Notre-Dame Press, 1981; Michael J. Sandel, Liberalism and the Limits of Justice, Cambridge, Cambridge University Press, 1982; Charles Taylor, « The Politics of Recognition » dans Amy Gutman (dir.), Multiculturalism and «The Politics of Recognition», Princeton, Princeton University Press, 1992, p. 25-74; Michael Walzer, The Spheres of Justice, New York, Basic Books, 1983; Amitai Etzioni, The Spirit of Community. New York, Crown Publishers, 1993; Michael J. Sandel, « Democrats and Community », The New Republic, 22 février 1988, p. 20-23. Je tiens à remercier Nicole Tremblay et Line Painchaud pour leurs nombreux et judicieux commentaires. 
récemment, Taylor propose en effet de revenir à un libéralisme moins militant, plus respectueux des désirs de la majorité et plus sensible aux besoins de la communauté, un libéralisme axé davantage sur l'idée de tolérance que sur celle de neutralitéz. Le libéralisme national a la québécoise, tel que vu et compris par Taylor, plus que le libéralisme multiculturel canadien, constituerait le modèle à suivre.

Plusieurs éléments de l'analyse et des propositions de Taylor me semblent soit laisser à désirer, soit complètement erronés. Premièrement, la distinction qu'il fait entre deux types de libéralisme n'est pas toujours claire. Deuxièmement, une fois la distinction précisée, le type de libéralisme que Taylor favorise n'est pas acceptable. Troisièmement, il est faux de prétendre que le Canada et le Québec adhèrent à des types différents de libéralisme.

\section{Deux libéralismes}

Dans son essai sur le multiculturalisme, Taylor distingue deux types de libéralisme ${ }^{3}$. Le premier ( $\mathrm{LI}_{1}$ reconnaît d'abord à « chaque citoyen un ensemble de libertés et de droits fondamentaux ». Ces droits et libertés reçoivent une protection absolue et ne peuvent varier selon le contexte culturel (p. 52). Ce type de libéralisme exige de plus que l'État, lors de ses inévitables et nécessaires interventions dans la vie économique, sociale et culturelle, prenne soin de « ne pas favoriser une conception du bien plutôt qu'une autre ». Une « société libérale est une société qui n'adhère à aucune vision substantielle des finalités de la vie humaine » et l'État qui doit incarner cet idéal ne doit, par conséquent, jamais se fixer de buts ou d'objectifs collectifs. Cette seconde exigence du premier type de libéralisme correspond à ce qu'il est maintenant convenu de nommer « la règle de neutralité de l'État $»(\text { p. } 56)^{4}$.

2. Charles Taylor, « The Politics of Recognition », dans Amy Gutman (dir.), op. cit; et « Convergences et divergences à propos des valeurs entre le Québec et le Canada » dans Rapprocher les solitudes. Ecrits sur le fédéralisme et le nationalisme au Canada,SainteFoy, Presses de l'Université Laval, I992, p. 179-214; et « Cross Purposes : The LiberalCommunitarian Debate », dans Nancy L. Rosenblum (dir.),Liberalism and the Moral Life, Cambridge, Harvard University Press, 1989, p. I59-182.

3. Charles Taylor, «The Politics of Recognition », dans Amy Gutman (dir.), op. cit. Les chiffres entre parenthèses renvoient tous à cet essai et les passages entre guillemets sont des traductions libres du texte de Taylor. Le texte original anglais sera cité lorsque cela s'avérera nécessaire. On trouvera les mêmes idées exposées dans les autres articles de Taylor cités à la note précédente.

4. Ceux qui les premiers ont employé le terme de « neutralité » ont maintenant pris une certaine distance vis-à-vis de l'expression, en partie pour les raisons que j'expliquerai plus loin. (Bruce A. Ackerman, « Neutralities», dans R. Bruce 
Dans une socièté libérale de type II, la cohésion sociale, qui ne peut se réaliser à partir d'un projet collectif ou d'une conception du bien, est « assurée par l'engagement de l'Etat à traiter tous les citoyens également » et par l'engagement de ces derniers à se respecter les uns les autres. « La raison pour laquelle l'État ne peut épouser une conception du bien » est que, ce faisant, il se « trouverait à désavantager ceux qui n'adhèrent pas à cette conception » et «violerait ainsi son engagement à traiter tous les citoyens également » (p. 5657).

Une des sources et des justifications les plus importantes de ce type de libéralisme est la reconnaissance de « l'autonomie des individus ». Les citoyens sont considérés « capables de décider eux-mêmes de ce qui est bon pour eux » et chacun d'eux doit en conséquence être autorisé à choisir pour lui-même son mode de vie, ses valeurs, etc. Cette vision du libéralisme a surtout pour origine « les pays de tradition politique anglo-américaine » et a récemment été « défendue par les philosophes Rawls, Dworkin et Ackerman » (p. 56).

Selon le second type de libéralisme (L2), « une société libérale peut adhérer à une conception du bien et poursuivre des buts et intérêts collectifs, sans que cela soit considéré comme une dévalorisation de ceux qui ne partagent pas ces objectifs ». Le seul fait que « la nature d'un bien demande qu'il soit poursuivi en commun constitue, pour les partisans de ce type de libéralisme, une raison suffisante de le faire devenir l'objet de politiques publiques ». Une société organisée selon L2 demeure cependant libérale par la « protection qu'elle accorde à certains droits et libertés fondamentaux et par la tolérance dont elle fait preuve à l'égard des minorités, surtout celles qui ne partagent pas les objectifs de la majorité » (p. 59).

Ce second type de libéralisme, plus interventionniste et plus sensible aux désirs de la majorité, est généralement justifié par l'importance du rôle de la communauté et de l'État dans le maintien de la stabilité sociale ainsi que dans la protection du cadre économique, social et culturel nécessaire au développement des individus.

Pour illustrer les différences existant entre ces deux types de libéralisme, ainsi que pour faire ressortir leurs forces et leurs faiblesses, Taylor utilise les cas du Canada et du Québec. Le Canada, par sa Charte des droits et libertés qu'il

Douglass, Gerard D. Mara et Henry S. Richardson (dirs), Liberalism and the Good, New York, Routledge, 1990, p. 29; Ronald Dworkin, A Matter of Principle, Cambridge, Harvard University Press, 1985. p. 191 et 205-213; Charles Larmore, Political Theory, vol. I8, n 3, août 1990, p. 341; John Rawls, Political Liberalism, New York, Columbia University Press, 1993, P. 191. Jutiliserai néanmoins cette expression parce que, malgré ses défauts, elle demeure la plus employée. 
souhaite voir appliquer à l'ensemble de son territoire, indépendamment du contexte culturel, et par sa volonté de demeurer neutre à l'égard des différentes conceptions du bien adoptées par ses citoyens, représenterait le premier type de libéralisme (p. 52-54). Le Québec, parce qu'il s'est donné pour objectif la survie et la promotion de la culture française, et parce qu'il est prêt à limiter l'exercice de certains droits et libertés reconnus par la charte canadienne pour atteindre son objectif, serait un représentant du deuxième type. La difficulté de concilier ces deux types de libéralisme constitue, selon Taylor, une des sources de l'impasse constitutionnelle canadienne (p. 52 et 59-61).

Entre ces deux conceptions du libéralisme, Taylor ne cache pas sa préférence pour la seconde. Selon lui, Li ne permet pas de protéger l'identité culturelle des peuples et des communautés (p. $\left.6 I^{5}\right)^{5}$.

\section{Irois différences?}

Étant donné que plusieurs éléments de la typologie que propose Taylor ne sont pas clairs, avant d'evaluer les mérites de chacune des deux formes de libéralisme il ne sera pas inutile de préciser ce qui les différencie vraiment. Les plus importantes différences pourraient se situer au niveau : a) du contenu de la liste des droits et libertés fondamentaux; b) du degré de protection qui leur est accordé; c) de la règle de neutralité de l'État.

a) Considérons d'abord les droits et libertés protégés par chacun des types de libéralisme. Du côté de L2, les droits et libertés qui seraient protégés sont : « le droit à la vie, la liberté, le droit à un procès équitable, la liberté d'expression, la liberté de religion, etc. » (p. 59). Le « etc. » est un peu gênant et j'y reviendrai.

En ce qui concerne la liste des droits et libertés protégés par LI, Taylor ne dit à peu près rien. Il laisse seulement sous-entendre que cette liste est beaucoup plus longue que celle protégée par L2 (p. 6I). Cette interprétation de Li est étonnante puisque la liste des droits et libertés de base à laquelle les théoriciens, que Taylor associe au premier type de libéralisme, désirent accorder une protection spéciale, bien que peut-être un peu plus longue que celle de L2, est généralement très courte. Elle comprend la plupart du temps les droits et libertés suivants : la liberté de pensée et de conscience, les libertés politiques (droit de vote, droit d'assemblée et de réunion), la protection contre la discrimination (sexe et race), le respect de l'intégrité physique et morale des

5. La classification et la position de Taylor sont endossées par Walzer dans son commentaire de l'essai de Taylor, « Comments on The Politics of Recognition », dans Amy Gutman (dir.), op.cit. 
citoyens et le droit de propriété personnelle (excluant les moyens de production) 6 .

Si on considère cette dernière énumération comme représentant la position standard des partisans de LI, ce qui distingue les deux listes de droits et libertès est donc que la liste de L2, favorisée par Taylor, ne mentionne ni les libertés politiques, ni la protection contre la discrimination, ni le droit de propriété personnelle.

Il y a deux façons d'interpréter la position et le texte de Taylor. On peut d'abord considérer que les droits et libertés qui ne figurent pas explicitement sur la liste de L 2 sont prises en compte par la locution « etc. ». Ou bien, on peut penser que Taylor ne considère pas ces droits et libertés comme fondamentaux. Dans le premier cas, les listes de Li et de Lz seraient identiques et il n'y aurait alors pas de différence à ce niveau entre les deux types de libéralisme. Dans l'autre cas, Lr et L2 différeraient certes substantiellement, mais on ne pourrait plus maintenir que Lz constitue une société libérale. A tout le moins, le type de libéralisme que Taylor favoriserait deviendrait beaucoup moins attrayant.

Pour les fins du présent exposé, adoptant l'interprétation qui me paraît la plus généreuse, je supposerai que les droits et libertés protégés par L2 sont les mêmes que ceux protégés par Lr et que la différence entre les deux types de libéralisme ne provient donc pas du contenu de la liste des droits et libertés de base.

b) Une seconde différence entre Lı et L2 pourrait se situer au niveau du degré de protection accordé aux droits et libertés fondamentaux. On pourrait penser que, selon Taylor, Li n'accepterait en aucun cas de compromettre la priorité des droits et libertés de base, tandis que L2 adopterait à cet égard une attitude plus souple. Cette interprétation justifierait les accusations « d'homogénéisation » que l'auteur porte contre $\mathrm{LI}^{7}$.

6. John Rawls, Théorie de la justice, Paris, Seuil, i987, ltr. fr. de A Theory of Justice, Belknap Press of Harvard University Press, 1971l, p. 92; Bruce A. Ackerman,Social Justice in the Liberal State, New Haven, Yale University Press, 1980, p. 177-178; Ronald Dworkin, «Foundations of Liberal Equality » dans The Tanner Lectures on Human Values conférence prononcée à l'Université Stanford, 5 et ro mai, 1988, p. 9. Contrairement à ce qu'insinue Taylor (p. 59), la protection de la langue et la liberté d'expression commerciale ne figurent jamais sur ces listes.

7. Les commentaires de Taylor sur les positions constitutionnelles du Québec confirmeraient cette lecture. Le Québec, société que Taylor prend comme exemple pour illustrer L2, serait prêt à limiter l'application de certains droits et libertés reconnus par la Charte canadienne des droits et libertés pour assurer la promotion de la culture française. 
La position que Taylor attribue explicitement à L2 est cependant, sinon conforme, du moins tout à fait acceptable pour les partisans de LI. Discutant la protection que L2 accorderait aux droits et libertés de base, Taylor écrit : «Those rights should never be infringed, and therefore ought to be unassailablyentrenched» (p. 59). Les partisans de Li n'en demandent pas davantage.

Taylor se trouve donc ici confrontè à un dilemme identique à celui discuté précédemment. Si L2 n'exige pas une solide protection des droits et libertés de base, par exemple du droit de vote ou de la liberté d'expression, le type de libéralisme ainsi créé, si tant est qu'il s'agisse encore de libéralisme, est difficilement acceptable. Si, cependant, L2 accorde une forte protection aux droits et libertés de base, il n'y a alors plus de différence significative sur ce point entre les deux types de libéralisme. Adoptant une fois de plus l'interprétation la plus généreuse, je supposerai que les deux types de libéralisme accordent une très grande protection aux droits et libertés de base précédemment énumérés.

c) Étant donné que la liste des droits et libertés de base ainsi que le degré de protection qui leur est accordé ne permettent pas de distinguer de façon significative les deux types de libéralisme, la distinction proposée par Taylor reposerait donc entièrement sur la règle de neutralité de l'État. L1 l'accepterait, tandis que L2 la rejetterait (p. 57). Commençons par préciser la signification et l'étendue de la règle de neutralité dèfendue par $\mathbf{L}$.

Taylor ne donne malheureusement pas beaucoup d'information concernant la règle de neutralité qu'il attribue à Lı, passant sous silence certaines distinctions fondamentales. Compte tenu de l'importance de la question, il faudra donc, à la place de Taylor, définir la règle de neutralité que défend LI.

Il existe deux interprétations de la règle de neutralité, la neutralité de conséquence et la neutralité de justification ${ }^{8}$. Selon la neutralité de conséquence, un Etat est neutre si ses interventions et ses politiques n'ont pas pour effet de favoriser une conception du bien plutôt qu'une autre ou de nuire

8. On trouvera des discussions des différents types de neutralité dans Robert $E$. Goodin et Andrew Reeve (dirs), Liberal Neutrality New York, Routledge, 1989; Peter De Marneffe, «Liberalism, Liberty, and Neutrality »,Philosophy and Public Affairs, vol. $20, n$ 2, 1991, P. I68-184; Will Kymlicka, « Liberal Individualism and Libera] Neutrality », Ethics, vol. 99, n" 4, I989. p. 884; Joseph Raz,The Morality of Freedom, Oxford, Clarendon Press, ig86, p. III-133; Charles Larmore, Patteris of Moral Complexity. Cambridge, Cambridge Universtiy Press, 1987, p. 42-45; William Galston, Liberal Purposes. Cambridge, Cambridge University Press, 1991, p. I00-1or; John Rawls, Political Liberalism, op. cit., p. 192-I94. 
à certains modes de vie. Tous les penseurs libéraux que Taylor associe à LI rejettent explicitement cette formulation de la règle de neutralité 9 .

Premièrement, les interventions de l'État, quelles que soient leurs justifications, ne peuvent s'empêcher de favoriser ou de défavoriser certaines conceptions du bien. Même la mise en application de la plus stricte neutralité de l'État ne peut éviter d'affecter différemment les modes de vie. Par exemple. la neutralité rend plus difficile la réalisation des conceptions du bien qui, pour s'épanouir et prospérer, exigent le soutien de l'État.

Deuxièmement, comme le reconnaissent les partisans de la neutralité de l'État, aucun type d'organisation sociale ne peut survivre s'il ne favorise pas les actions qui sont nécessaires au maintien de l'organisation ou, du moins, s'il ne décourage pas les actions qui risquent de miner son fonctionnement. À cet égard, les sociétés libérales de type Li ne sont pas plus suicidaires que les autres organisations sociales. Comme les autres sociétés, les sociétés libérales considèrent que leur projet de société constitue, sinon la meilleure et la plus morale, du moins une forme très valable d'organisation sociale, et autorisent en conséquence l'État à prendre parti en faveur d'une conception du bien si cela est la seule façon de permettre aux institutions libérales de survivre et de prospérer. Par exemple, le maintien des institutions libérales peut exiger des règles de mariage et de divorce, une répartition des richesses plus égalitaire, une politique de natalité et d'immigration, une politique d'éducation, une politique linguistique et culturelle, une politique industrielle, une politique de l'environnement, dans plusieurs cas le financement minimal des arts et des sciences, etc.

Ce que propose les libéraux ( $\mathrm{L}_{\mathrm{I}}$ ), ce n'est donc pas la neutralité de conséquence, mais la neutralité de justification. Selon cette définition de la neutralité, ce ne sont pas les conséquences des interventions de l'État qui doivent être neutres vis-à-vis des diverses conceptions du bien, mais les raisons invoquées par l'État pour justifier ses interventions. L'État ne doit pas chercher à favoriser une conception du bien plutôt qu'une autre ou avoir lintention d'aider ceux qui poursuivent un but en particulier. Ses interventions et politiques ne doivent aucunement être motivées par le désir de faire la promotion d'un mode de vie ou par la volonté de favoriser les intérêts d'un groupe en particulier. L'État ne doit intervenir que lorsqu'il est motivé par une justification neutre; рат exemple, pour maintenir la stabilité sociale, stimuler la productivité, garantir la sécurité nationale, assurer la survie des institutions libêrales, etc.

9. Bruce A. Ackerman, « Neutralities », dans R. Bruce Douglass et al. (dirs),op. cit., p. 38-39; John Rawls, op. cit., p. 193; Will Kymlicka, loc. cit., p. 884; Charles Larmore, Patterns of Moral Complexity, op. cit., p. 44. 
La règle de neutralité défendue par les partisans de Li n'implique donc pas du tout la non-intervention de l'État. Tant que les justifications utilisées à l'appui des politiques proposées demeurent neutres, la règle de neutralité de justification peut autoriser de substantielles interventions de l'État dans l'organisation de la vie économique, sociale et culturelle.

Même en définissant aussi largement que nous venons de le faire la règle de neutralité défendue par Lı, il n'y a pas de doute qu'il existe ici une différence majeure entre les deux types de libéralisme étudiés par Taylor. Le premier, même s'il n'exige pas la non-intervention de l'État oula neutralité des conséquences de ses actions, impose néanmoins des limites strictes à son intervention. Le second type de libéralisme n'impose quant à lui que très peu de limites au pouvoir de l'État. Mis à part la protection de certains droits et libertés de base et le respect des minorités, l'État conserve, comme nous le verrons à l'instant, une marge de manœuvre considérable (p. 59).

Ayant identifié ce qui distingue essentiellement les deux types de libéralisme, il s'agit maintenant de prendre position.

\section{L2 et l'intervention de l'ttat}

Les principales difficultés que soulèvent chacun des deux types de libéralisme sont clairement identifiées par leurs adversaires ${ }^{10}$. Pour ceux qui défendent LI, le libéralisme interventioniste que propose L2 menace les droits et libertés de la personne. Pour les partisans de $\mathrm{L} 2$, la règle de neutralité favorisee par Li ne tient pas assez compte des différences culturelles et force l'homogénéisation des sociétés.

Contrairement à Taylor, je suis d'avis que la menace représentée par L2 est plus sérieuse que l'autre. La règle de neutralité défendue par li me semble mieux circonscrire les interventions de l'État et je pense être en mesure de répondre aux craintes de Taylor concernant l'homogénéisation des sociétés.

Comme il a déjà été mentionné, le libéralisme que favorise Taylor n'impose que très peu de limites à l'intervention de l'État. Dans la mesure où les sociètés demeurent tolérantes envers leurs minorités et respectent certains droits et libertés fondamentaux, elles peuvent favoriser des conceptions du bien et encourager des modes de vie auxquels n'adhèrent pas plusieurs de leurs membres. Comme l'écrit Taylor, « le seul fait que la nature d'un bien demande qu'il soit poursuivi en commun, constitue une raison suffisante de le faire

10. Voir les commentaires de Michael Walzer dans Amy Gutman (dir.), op. cit., ainsi que ceux de Steven C. Rockefeller, « Comments on The Politics of Recognition », dans Amy Gutman (dir.), ibid., p. 87-98. 
devenir l'objet de politiques publiques » (p. 59). Nommons la règle de L2 gouvernant les interventions de l'État, la règle de tolérance.

Taylor ne mentionne pas comment sera décidé quelles conceptions du bien seront encouragées ni quels biens seront recherchés. L'omission est malheureuse parce que le choix de la procédure de décision déterminera dans une large mesure le caractère de la société. On peut cependant penser qu'en tant que partisan de l'humanisme civique ${ }^{\mathrm{II}}$, Taylor désire, comme d'ailleurs d'autres

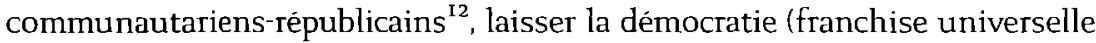
et règle de majorité) trancher ces questions. Cette interprétation est la seule acceptable si Taylor entend réellement défendre une position libérale, toutes les autres options consistant à imposer autoritairement une conception du bien.

Quelle que soit cependant la méthode retenue pour décider des interventions de l'État, la règle de tolérance proposée par Taylor et les partisans de L2 est de toute évidence trop permissive et, dans une certaine mesure, contraire à l'esprit de la philosophie libérale. Premièrement, la règle ne respecte pas certains de nos jugements moraux bien pesés et va à l'encontre de pratiques libérales solidement établies. Par exemple, cette règle rejette la séparation de l'Église et de l'État. En effet, la règle de tolérance permet à l'État, tant que celui-ci n'empêche pas les individus de pratiquer la religion de leur choix. de soutenir et de favoriser financièrement une religion particulière.

Deuxièmement, la règle peut rendre très difficile l'exercice des droits et libertés fondamentaux. Plus précisément, la règle repose sur la distinction entre ce que l'État peut interdire et ce qu'il peut favoriser, entre l'incitation et la coercition. Tant que l'État n'empêche pas les individus d'exercer leurs libertés de base, l'État demeure libre d'utiliser les mesures qu'il juge appropriées pour faire la promotion d'une conception du bien. Cette distinction entre interdire et favoriser ne garantit cependant pas suffisamment la protection des droits et libertés de base des individus, car les moyens que peut utiliser l'Etat pour encourager certaines conceptions du bien sont si puissants qu'ils risquent de diminuer considérablement la portée réelle des droits et libertés des individus. Prenons par exemple le cas de l'éducation. Si l'État ne subventionnait qu'un type d'éducation confessionnelle, les coûts élevès du maintien d'un système parallèle non confessionnel ou d'une confession différente pourraient constituer un empêchement majeur au plein exercice de la liberté de religion. La frontière entre moyens incitatifs et

II. Charles Taylor, Philosophical Papers, vol. 3. Cambridge, Cambridge University Press, 1985, p. 334 .

12. Voir Michael Sandel, « The Procedural Republic and the Unencumbered Self », Political Theory, vol. 12, 1984. 
coercitifs est si ténue et peut être franchie avec tant de facilité qu'une règle plus stricte que celle proposée par Taylor est requise pour protéger efficacement les droits et libertés de base des citoyens contre l'intervention de l'État.

Troisièmement, la règle ne protège pas suffisamment les droits et libertés secondaires (la liberté de se marier et de divorcer, la liberté de choisir son occupation et ses loisirs, etc.). Ce n'est pourtant pas parce que ces libertés ne peuvent jouir d'une protection aussi absolue que les droits et libertés de base. qu'elles nous sont indifférentes ou que nous sommes prêts à laisser la majorité, sans limite, décider de leur sort.

Pour ces raisons, la règle de tolérance proposée par L2 n'est pas acceptable $\mathrm{d}^{\prime} u n$ point de vue libéral ${ }^{13}$. La règle de neutralité de justification, parce qu'elle permet de mieux contenir les interventions de l'État, est beaucoup plus conforme à la tradition libérale qui, rappelons-le, est d'abord animée par la volonté de limiter le pouvoir de l'État ${ }^{14}$. La règle de neutralité n'est bien sùr pas parfaite. Dans certains cas la règle n'offre qu'une ligne de conduite assez vague et dans plusieurs autres il peut s'avérer difficile d'identifier les motivations réelles des acteurs $^{15}$. Malgré ces défauts, parce qu'elle impose au moins une limite à l'intervention de l'État, la règle de neutralité de justification me semble constituer une position de départ plus prudente et plus acceptable que la règle de tolérance proposée par L2. Risque-t-elle cependant de forcer l'homogénéisation des sociétés?

\section{L1 et l'homogénéisation}

Pour illustrer le danger d'homogénéisation que fait courir aux sociètés l'adoption de LI, Taylor soutient qu'une adhésion stricte à la règle de neutralité de l'État empêcherait les sociétés libérales de faire la promotion, et même d'assurer la survie, de la culture d'une nation, d'un peuple ou d'une commu-

13. Le libéralisme, comme le rappelle fort justement Judith N. Shklar dans « The Liberalism of Fear », dans Nancy L. Rosenblum (dir.), op.cit., p. 23, c'est plus que de la simple tolérance.

I4. La première phrase du célèbre essai sur la liberté de J. S. Mill (On Liberty), le père du libèralisme moderne, est la suivante : «The subject of this essay is not the so-called 》 liberty of the will, "so umfortumately opposed to the misnamed doctrine of philosophical necessity; but civil, or social liberty: the nature and limits of the power which can be legitimately exercised by society over the individual s (Indianapolis, Hackett, 1978). Voir aussi les commentaires pertinents de Charles Larmore, « Political Liberalism », op. cit., p. 339; et de Judith N. Shklar, loc. cit., dans Nancy L. Rosenblum (dir.), au sujet de la raison d'être du libéralisme.

15. Sur les difficultés soulevées par la règle de neutralité de justification, on peut consulter William A. Galston, op.cit, p. IOz. 
nauté. Parce que ces sociétés s'interdiraient de promouvoir une conception du bien, elles seraient dans l'impossibilité de protéger leur identité culturelle. A plus ou moins long terme, les cultures incapables de soutenir la compétition internationale ou nationale seraient graduellement assimilées, entraînant pour leur membres les difficultés reliées à la perte de son identité culturelle. Par exemple, Taylor prétend que l'adhésion à la règle de neutralité de l'État enlèverait au Québec les outils nécessaires à la défense et à la promotion de la culture française, compromettant ainsi son avenir (p. 6o).

La critique de Taylor me paraît excessive et une meilleure connaissance des implications sociales et culturelles de la règle de neutralité de justification devrait calmer les principales inquiétudes des libéraux-communautariens. Je commencerai par distinguer deux groupes de peuples ou de communautés qui pourraient se sentir menacés par l'action homogénéisante de la règle de neutralité : I) les peuples constitués en États-nations qui désirent protéger leur culture de la compétition internationale ou des puissantes cultures avoisinantes (Finlande, Norvège, Lituanie, etc.); 2) les peuples intégrés à un plus grand ensemble, menacés par la culture ou l'ethnie dominante ${ }^{16}$ (les minorités nationales et les peuples autochtones ${ }^{17}$ ).

En ce qui concerne les États-nations, les mesures adoptées par certains États pour protéger leur culture de l'attraction de cultures voisines plus fortes peuvent facilement être justifiées sans enfreindre la règle de neutralité de justification ${ }^{18}$. Dans un contexte de globalisation des marchés, d'ouverture des

I6. Le Québec peut faire partie de la première ou de la seconde catégorie, selon qu'on le considère comme un Etat souverain ou simplement comme une province. Je reviendrai plus loin sur l'ambiguïté de l'analyse de Taylor concernant cette question.

17. Dans les discussions qui suivent, il sera exclusivement question des cultures et ethnies nationales (autochtones, nations conquises, etc.), et donc pas du tout des communautés ethniques fondées sur l'immigration (Italiens de Montréal. Irlandais de Boston, etc.). Sur cette distinction, on peut consulter Will Kymlicka, «Liberalism and the Politicization of Ethnicity », Collective Rights, The Canadian Journal of iaw and Jurisprudence, vol. IV, $\mathrm{n}$ 2, 199r; Chandran Kukathas « Are There Any Cultural Rights? », Political Theory, vol. 20, n" I, 1992; et John Danley, « Liberalism, Aborigenal Rights and Cultural Minorities », Philosophy and Public Affairs, vol. 20, $n^{\prime \prime}$ 2, 1991, p. 253-274.

I8. Il n'est pas inutile de rappeler que les deux plus grands théoriciens de la neutralité de l'Etat, Kant et Rawls, n'ont jamais souhaité la disparition de l'Etat ni demandé l'abolition des frontières. Bien au contraire, ils se sont clairement prononcés contre la création d'un gouvernement planétaire. Voir Kant, Idée d'une histoire universelle au point de vue cosmopolitique, Paris, Bordas, 1988, et John Rawls, « The Domain of the Political and Overlapping Consensus $\gg$ New York University Law Review, vol. 64, n' 2, rg89, p. 251-252, note 46. 
frontières et d'internationalisation des communications et des arts, la protection d'une culture nationale, l'établissement d'une ou de plusieurs langues officielles ainsi que la mise en place de politiques d'immigration constituent à n'en pas douter des mesures nécessaires à l'épanouissement des institutions libèrales. Sans ces mesures, il deviendrait en effet très difficile de maintenir un espace public homogène garantissant la stabilité des sociétés et permettant la tenue d'un dialogue véritable entre les membres de la communauté ${ }^{19}$. Autant de justifications qui demeurent neutres à l'égard des différentes conceptions du bien des citoyens ${ }^{20}$.

L'ensemble de ces politiques culturelles protectionnistes doivent, bien entendu, demeurer dans les limites du raisonnable. Elles doivent être adaptées à la situation de chaque pays, répondre à des menaces réelles et ne constituer que le minimum nécessaire au maintien de la stabilité sociale et à la sauvegarde des institutions libérales. Ces mesures peuvent être comparées aux autres politiques sociales (éducation, natalité, promotion des arts et des sciences, etc.) que tous estiment nécessaires au bon fonctionnement d'un État libéral et démocratique moderne. Que la plupart des pays, y compris les sociétés qui selon Taylor adhèrent à la règle de neutralité de l'État, aient adopté de telles mesures, témoigne de leur nécessité. L'adhésion à la règle de neutralité de justification n'empêche donc nullement les peuples de protéger leurs culture $^{2 \mathrm{I}}$.

Anticipant cette réponse, Taylor réplique que la protection ou la promotion d'une culture ne constituent pas des objectifs neutres comparables à la protection des espaces verts ou à la purification de l'air (p. 58 et 40 note i6). La culture n'est pas un bien qui est neutre à l'égard de toutes les conceptions du bien, donc un bien nécessaire à la réalisation de tous les projets de vie, mais constitue plutôt une conception du bien en soi. En faisant la promotion d'une culture, l'État favorise la conception du bien de ceux qui adhèrent à cette culture au détriment de ceux qui veulent choisir leur langue et leur culture.

La réplique de Taylor révèle une sous-estimation, assez curieuse pour un communautarien, de la contribution de plusieurs composantes de l'environnement culturel à la réalisation du projet de vie ou de la conception du bien de

19. J.S. Mill considèrait l'existence d'une langue commune comme une condition de la démocratie. Cité par Yael Tamir, Liberal Nationalism. Princeton, Princeton University Press, 1993, p. 40.

20. De telles mesures sont d'autant plus nécessaires lorsqu'il s'agit de « petites cultures ", comme la Norvège, la Lituanie ou le Québec.

21. Dans cette perspective, les mesures linguistiques et les politiques de l'immigration adoptées par le gouvernement du Québec me paraissent. sinon justifiées, du moins demeurer dans les limites du justifiable. 
chaque citoyen. Tous les membres de la société, y compris les immigrants, ont intérêt à ce que la culture de la société dont ils font partie soit stable et vigoureuse, que l'information circule rapidement et que les citoyens possèdent un minimum de références culturelles, artistiques et scientifiques communes.

\section{L1 et L2 et la protection des minorités ethniques et culturelles}

La justification de certaines mesures de protection culturelle à l'échelle nationale ne semble donc pas poser plus de difficultés au libéralisme de type Lı qu’à celui de type $L$ z. Le véritable défi auquel fait face le libéralisme, quel que soit son type, est plutôt de trouver un moyen de justifier la protection des cultures ou ethnies minoritaires menacées par une communauté ethnique ou par une culture nationale plus forte. D'une part, la nécessité de maintenir un espace public homogène autorise la société ou le groupe majoritaire à imposer certaines normes culturelles ou linguistiques à l'ensemble de son territoire. D'autre part, le bien-être et le développement harmonieux des individus membres de cultures minoritaires passent dans bien des cas par la reconnaissance et la protection de leur culture. Comment réconcilier ces deux considérations? Est-ce que L2 offrirait une solution plus acceptable que Li à ce problème?

Du côté de Li la question est de savoir si la règle de neutralité de justification autorise la mise en place de mesures spéciales pour assurer la protection des minorités culturelles. Deux justifications se prétendant neutres ont été suggérées. La première fonde la défense des droits des minorités ethniques et culturelles sur l'histoire et l'obligation de réparer le tort causé à ces peuples $^{22}$. L'autre, moins traditionnelle, revendique pour les minorités la reconnaissance d'un droit à la culture en insistant sur l'importance de l'environnement culturel dans le développement de la personnalité de l'individu ${ }^{23}$. Ces deux justifications possèdent leurs forces et leurs faiblesses. La première est bien ancrée dans nos traditions et nos mœurs politiques, mais offre peutêtre une protection trop limitée. La réparation des torts causés n'exigent pas nécessairement la création de droits ou de privilèges. La seconde justification semble mieux en mesure d'assurer une protection à long terme des minorités

22. Cette position est deffendue, entre autres, par D. Sanders, « The Re-Emergence of Indigenous Questions in International Law », Canadian Human Rights Yearbook Toronto, Carswell, r983; et John R. Danley, loc. cit. Pour d'autres références, voir Danley, ibid., p. I83 note 8; et Will Kymlicka, Liberalism, Community and Culture, Oxford, Clarendon Press, i99i, p. 153 .

23. Will Kymlicka, ibid., (chapitre 8). On trouvera une discussion de la position de Kymlicka chez Chandran Kukathas loc. cit; John R. Danley, loc. cit; et Yael Tamir, Liberal Nationalism, op cit. 
ethniques et culturelles. Elle risque cependant de justifier l'attribution de droits et de privilèges à un trop grand nombre de groupes minoritaires, par exemple aux immigrants.

Je ne commenterai pas davantage le bien-fondé ni la neutralité de ces justifications, me contentant de renvoyer aux nombreuses discussions qu'elles ont suscitées $^{24}$. Je les ai mentionnées surtout pour illustrer les ressources de la régle de neutralité de justification et montrer comment le libéralisme de type Li peut répondre aux préoccupations légitimes des minorités ethniques et culturelles.

Du côté de L2, les garanties offertes aux minorités ne sont pas très fortes, à tout le moins pas plus solides que celles fournies par LI. D'abord, la quasiabsence de limite imposée à l'intervention de l'État en faveur de la conception du bien de la majorité fait peser de façon permanente une menace sur les minorités. L2 ne prévoit en effet à peu près aucune mesure pour arbitrer les conflits entre un groupe majoritaire et une culture minoritaire. En ce qui concerne le devoir de tolérance à l'égard des minorités, seule limite imposée par L2 aux désirs de la culture dominante, son effet ne pourra être évalué que lorsqu'on saura ce que signifie réellement cette idée de « tolérance ». Étant donné la réticence de Taylor et des communautariens en général à multiplier les droits et libertés, il serait étonnant que cette idèe signifie autre chose que la reconnaissance d'un droit à l'association, droit que reconnaît également le libéralisme de type $\mathrm{Li}^{25}$.

Au chapitre de la protection et du respect des minorités ethniques et culturelles, le libéralisme de type Li offre donc des garanties aussi, sinon plus, solides que le libéralisme de type L2.

En conclusion des deux sections précédentes consacrées au danger d'homogénéisation que ferait courir aux sociétés la règle de neutralité de l'État, il faut reconnaître que la menace est loin d'être aussi sérieuse que Taylor le prétend.

\section{Le Québec et le Canada}

Comme je l'ai fait remarquer lors de ma présentation de l'analyse de Taylor, ce dernier, après avoir associé le Canada au premier type de libéralisme et le Quebec au second, attribue une partie des difficultés actuelles de la fédération canadienne à l'incompatibilité des deux types de libéralisme.

24. Voir les trois notes précédentes.

25. Pour une critique des positions communautariennes à l'égard des minorités, voir Will Kymlicka, op. cit, (chapitres iा-т2). 
Avant d'entreprendre cette démonstration, signalons que le choix d'un Etat (le Québec) ne possédant pas l'exclusivité du pouvoir sur son territoire pour illustrer l'un des deux type de libéralisme (L2) n'est pas très heureux. Certaines des interventions de l'État québécois ne s'expliquent en effet uniquement qu'en réaction aux interventions de l'État canadien et ne révèlent par conséquent pas nécessairement sa vraie nature. Pour illutrer L2, il aurait été préférable d'utiliser un pays souverain et indépendant dont la culture est menacée, par exemple la Norvège.

Pour évaluer l'analyse de Taylor, il faudra donc faire abstraction de cette irrégularité et considèrer le Québec comme un État souverain. Ce petit exercice de politique fiction, que le lecteur canadien n'aura aucune peine à accomplir, permettra de montrer que le Québec et le Canada adhèrent tous les deux au premier type de libéralisme.

Premièrement, comme en témoignent leurs Chartes des droits et libertès, le Québec et le Canada acceptent tous les deux la liste de droits et libertês de base proposée par les partisans de $\mathrm{L}$ : la liberté de pensée et de conscience, le droit de vote, le droit d'assemblée et de réunion, la protection contre la discrimination, le respect de l'intégrité physique et morale des citoyens et le droit de propriété personnelle ${ }^{26}$. Le différend qui oppose les deux sociétés en matière de droits et de libertés individuels concerne uniquement quelques applications des droits linguistiques. Ces droits ne figurent cependant jamais sur la liste des droits et libertés de base protégés par LI. Tout simplement parce que même si les partisans de Ir reconnaissent l'importance des droits linguistiques, l'extrême diversité des situations nationales empêche de leur accorder une protection absolue ${ }^{27}$.

Deuxièmement, toujours se référant à leurs Chartes des droits et libertès, on peut constater que le Canada et le Québec accordent tous les deux une très grande protection aux droits et libertés de base. Le Québec ne désire se soustraire qu'à certaines dispositions linguistiques de la Charte canadienne.

Troisièmement, les deux sociétés peuvent facilement justifier l'intervention de leur État dans l'organisation de la vie économique, sociale et culturelle par la règle de neutralité de justification. Le Québec n'est à cet égard pas plus interventionniste que le Canada. Il peut justifier l'adoption de

26. Gouvernement du Quêbec, Charte des droits et libertés de la personne, L.R.Q., lchapitre C-ı2l, Editeur officiel du Québec, 1993. Gouvernement du Canada, Charte canadienne des droits et libertés, lannexe B, partie I de la loi constitutionnelle de I982l, ministère de la Justice du Canada, ig89.

27. Même si on concédait que les droits linguistiques doivent être considérés comme des droits de base, la différence entre les libéralismes pratiqués par le Canada et le Québec serait si minime qu'elle ne justifierait pas l'analyse de Taylor. 
certaines mesures culturelles protectionnistes en invoquant la nécessité de protéger la vitalité des institutions libérales et de favoriser l'épanouissement des libertés fondamentales de ses citoyens, tout comme le fait le canada pour justitier ses propres mesures economiques, sociales et culturelles protectionnistes $^{28}$. En fait, le Québec ne revendique rien de plus que ce que le Canada et toutes les démocraties libérales possèdent déjà, à savoir une reconnaissance internationale, une politique culturelle, une politique des langues officielles et une politique d'immigration.

Les deux sociétés, le Québec et le Canada, partagent donc la même conception du libéralisme. L'impasse constitutionnelle canadienne ne s'explique alors pas par l'affrontement de deux types de libéralisme, mais plutôt, comme le veut une explication plus traditionnelle, par les difficultés soulevées par l'intégration de deux peuples qui, bien que profondement attachés aux valeurs libérales et démocratiques, sont confrontés à des situations dëmographiques et linguistiques différentes. Les Canadiens anglais sont nombreux et majoritaires, n'ont aucune raison de craindre pour leur langue, mais s'inquiètent des effets de la puissante force d'attraction de la culture américaine. Les Canadiens français sont moins nombreux et minoritaires, leur langue est menacée, mais leur identité nationale est bien définie.

\section{Conclusion}

De l'ensemble de la discussion précédente, il faut retenir les trois points suivants. Premièrement, la différence essentielle entre les deux types de libé ralisme étudiés par Taylor réside dans la règle gouvernant les interventions de l'État. Le premier autorise l'État à intervenir dans l'organisation de la vie économique, sociale et culturelle d'une société uniquement lorsque ces interventions peuvent être justifiées de façon neutre. Le second type de libéralisme n'impose que très peu de limite à l'intervention de l'État. Il demande seulement que l'État fasse preuve de tolérance à l'égard des minorités.

Deuxièmement, il faut favoriser le premier type de libéralisme. Le second ne parvient pas à contenir les interventions de l'État dans les limites du raisonnable et ne respecte donc pas l'esprit de la tradition libérale. La règle de neutralité de l'État proposée par li (neutralitê de justification), bien qu'imparfaite, garantit l'exercice des droits et libertés de base et assure une protection effi cace des libertés secondaires. Raisonnablement interprétée, elle autorise l'adoption de mesures culturelles protectionnistes a l'échelle nationale et justifie la protection des minorités ethniques et culturelles.

28. Voir la clause du traité de libre-échange Canada/Etats-Unis qui place hors de juridiction de l'entente, l'édition, le disque et les activités audiovisuelles. 
Troisièmement, l'impasse constitutionnelle canadienne ne doit pas être interprétée comme l'affrontement de deux types de libéralisme, mais plutôt comme les difficultés d'intégration de deux peuples partageant sensiblement les mêmes valeurs. Le Québec n'est pas moins libéral que le Canada et ce dernier est tout aussi nationaliste que le Québec.

La distinction que Taylor établit entre les deux types de libéralisme n'apporte donc aucun éclairage nouveau sur la nature du libéralisme ou sur les relations entre le Québec et le Canada.

Département de philosophie Cegep d'Ahuntsic 